\section{The Life and Death of a Tungsten Hairpin Filament}

\author{
Steve Chapman \\ Senior Consultant, Protrain \\ protrain@emcourses.com
}

Many words have been written about filament saturation and filament life, not all with years of experience behind them! Sometimes when visiting laboratories one has the feeling that the life of the filament is far more important than the quality of the results, so let us try to demystify filament life?

The life of a tungsten hairpin filament very much depends upon its use and the applications present in a particular laboratory. Medium to low magnification applications in the transmission electron microscope (TEM) may only require low emission currents (10 to 15 micro amps) with a long filament life resulting. High resolution studies require higher operating currents (20 to 45 micro amps) but, by demanding more emission current, the life of the filament will suffer. Whilst a long filament life is good for the laboratory, it is not worthy of boasting, for those who have a short filament life may well be using the instrument near to the limit of its performance, a far more relevant boast!

Similar situations in filament life relate to the scanning electron microscope (SEM). Where a hundred plus hours may be possible in a TEM, used at low magnification levels $(<30,000 X)$, one would expect 60 to 70 hours in a SEM used at low levels of magnification $(<5,000 X)$ or for analytical work. At high resolution, or at low accelerating voltages and constantly pushing the instrument to its limit, SEM filament life may well be only 15 to 20 hours.

So why do we have these differences? It's all about filament position and emission. The more emission one demands from the filament the hotter it needs to be to reach saturation, this results in a higher level of oxidation and evaporation and a shorter filament life. However, whilst one cannot have something for nothing, it is possible to obtain even more from the filament if it is run hard. Diagram 1 demonstrates the change in performance of a typical SEM when the filament to cathode cap position is varied. As resolution is improved, filament life is lost, but through optimisation it is possible to make a spectacular change to most SEM's performance. Make similar changes to a TEM gun, through shorter filament to cathode distances, and there is the opportunity for smaller apertures in the condenser system and smaller spot sizes, which enables higher

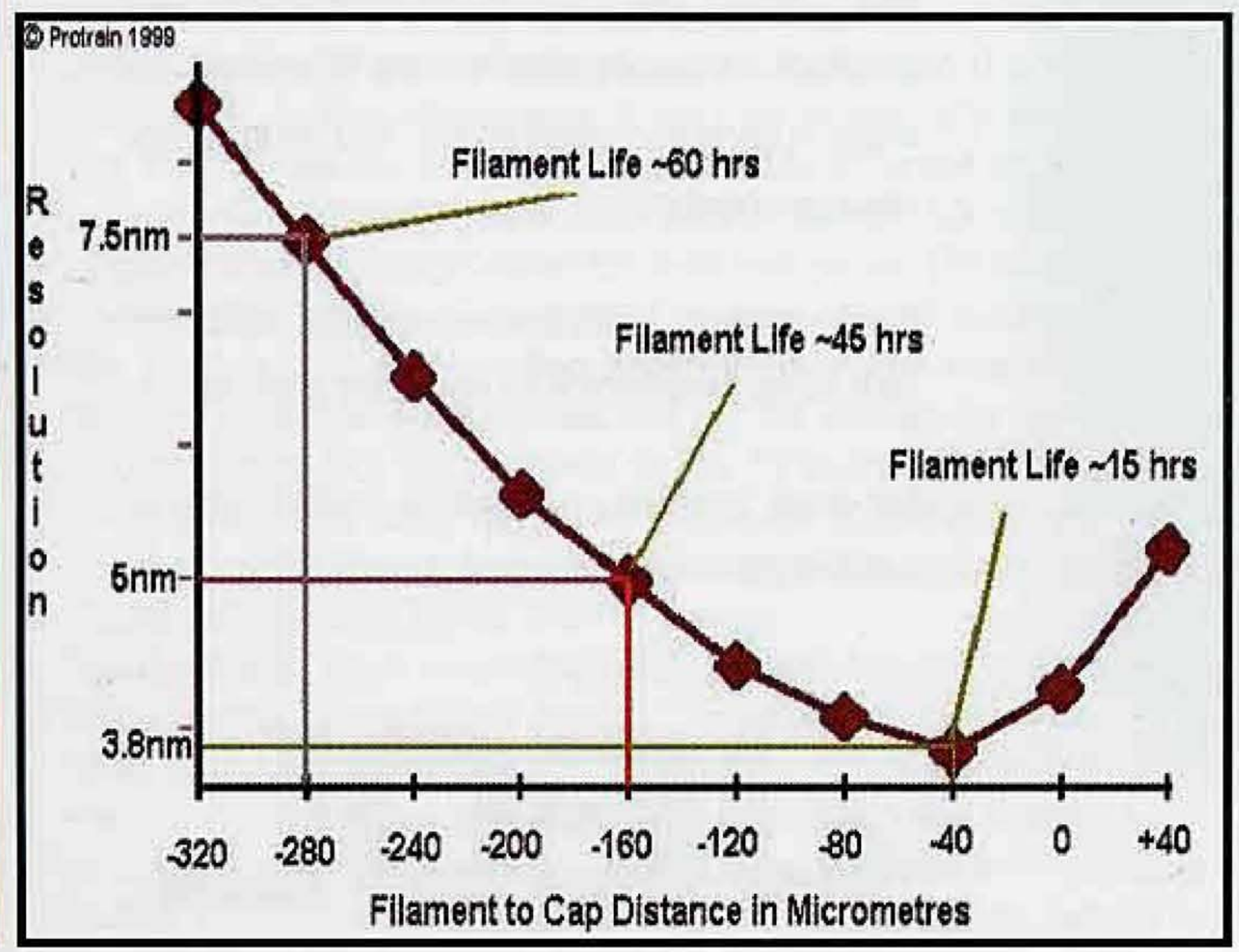

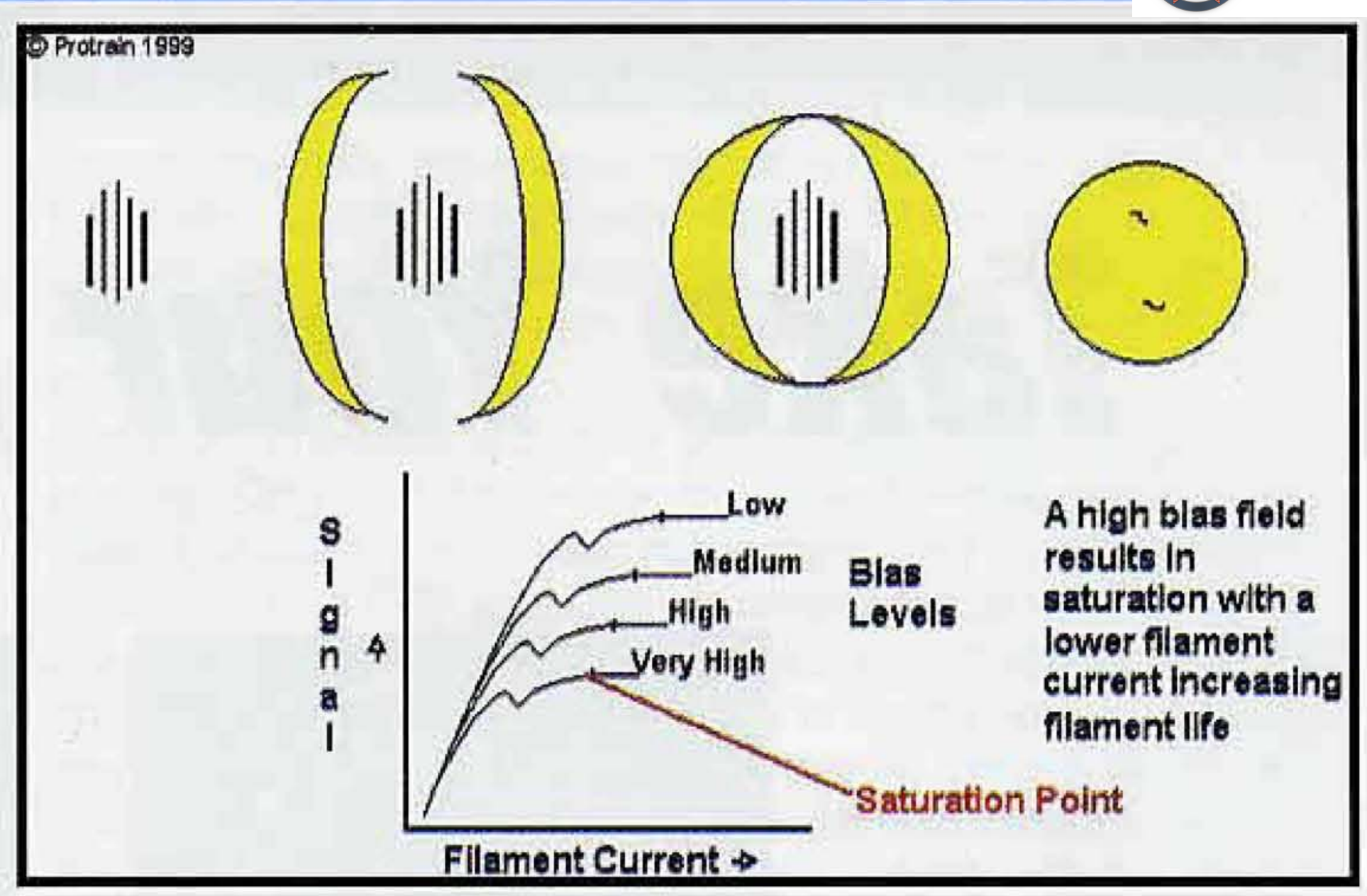

image coherency and a visible improvement in image quality.

So how does one saturate the filament? I believe we would all understand this procedure a good deal better if we called it "saturating the cathode," because that is what we are doing. Most TEM operators heat the filament whilst watching the image of the virtual source that we see at condenser crossover (Diagram 2). Many believe that we need to oversaturate, or overheat the filament, to run on the flat part of the graph (Diagram 2). The original reason for this was to compensate for filament supply instabilities, I believe this is not necessary today. Take a look at the virtual source of a modern TEM at 50,000X undersaturated, watch it for some time, if the instrument is working correctly it will not change at all; who needs to oversaturate? As an ex-demonstrator, but still a TEM operator, I do not saturate completely, I leave just a few dark spots in the source for a deliberate reason. If the operator is able to see the edge of the beam as it is brought to condenser crossover it is easy to see the crossover condition. But, higher up the magnification range, it is not possible to see the edge of an aligned beam-so one must utilize the dark patches that undersaturating the filament places on the beam image as an excellent signal of condenser cross over. Why is this so important? High coherence is almost always a TEM operator's objective and the best way to do this is to run the condenser system in an overfocus condition, turn clockwise from condenser crossover!

In order to obtain any level of performance from a hairpin filament in an SEM, one needs to saturate the filament fully. It is no good running at the first peak as the source is too large and the operator sacrifices too much probe current using the condenser lenses to obtain a suitable sized probe for high performance. Once again there is no substitute for what I believe are the correct emission current figures which are set out here in Table 1, below. The "correct emission" is attained either through bias adjustment, or in an ideal situation, via moving the filament forward with the bias in

W Hainpin Systems
ABT, ISI, Topcon $=100$ microamps $+\mathrm{kV}$
Camscan $=120$ microamps
Hltachl $=100$ microamps
JEOL $=100$ microamps $+\mathrm{kV}$
LEO, Cambridge $=400$ microamps
FEI, Phillps $=50$ microamps
LaB ${ }_{6}$ settings tend to be about one quarter to one half of these values
FEG systems run between $B$ and 20 microamps



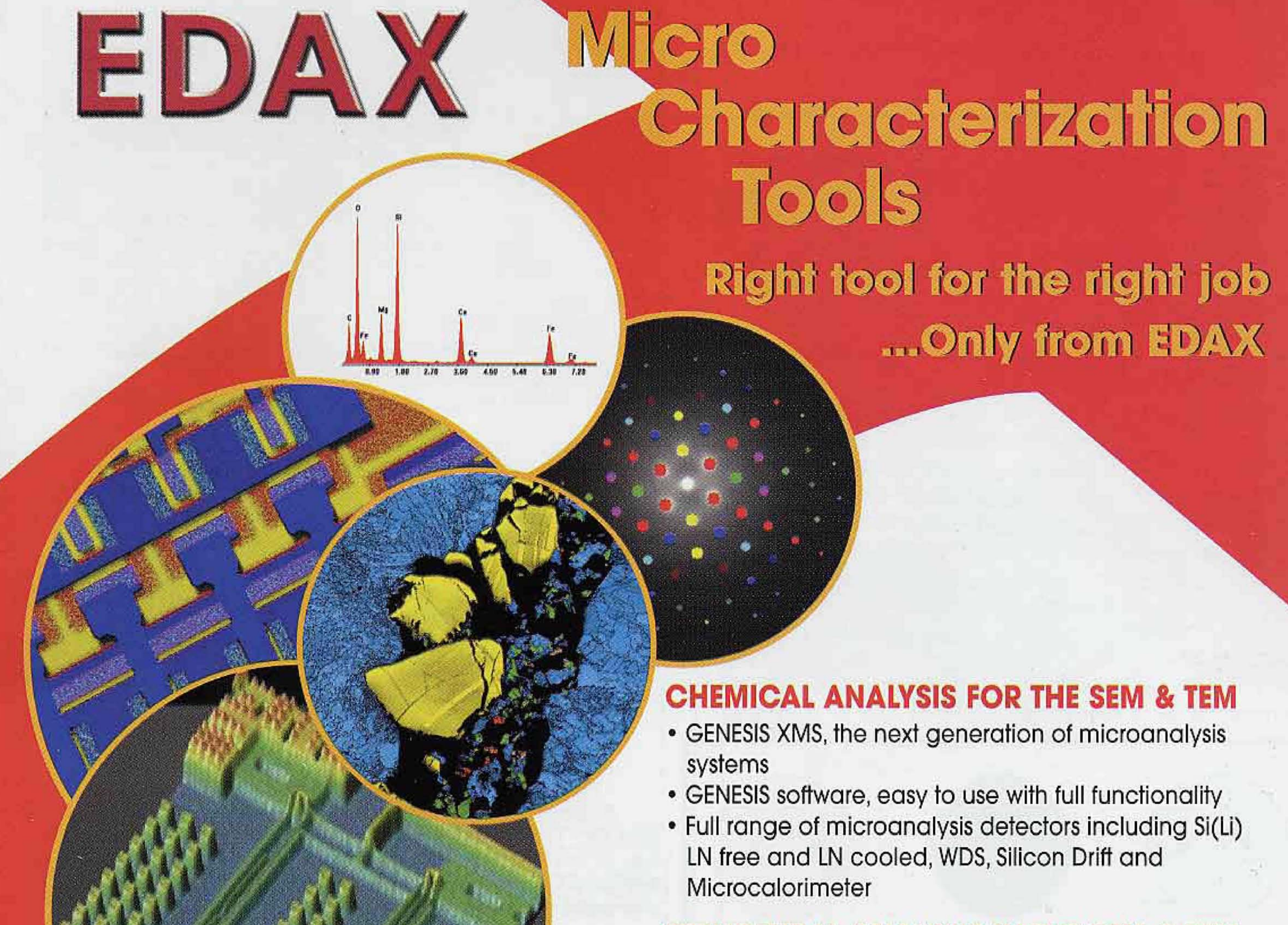

\section{STRUCTURAL ANALYSIS IN THE SEM \& TEM}

- EBSD in the SEM

OIM provides crystal orientation mapping and texture analysis

Delphi combines EBSD and EDS data for phase identification

- Electron Diffraction in the TEM

$A C T$, automated tools for the acquisition, analysis and reporting of TEM diffraction data

\section{INTEGRATED SOLUTIONS}

- Take micro characterization to the next level and achieve the most accurate results with simultaneous EDS/EBSD data acquisition and Chl-Scan chemical indexing

Americas 201-529-4880

Japan $+81-3-3740-5+72$

Europe, Africa and Middle East

$+31-13-536-4000$

S.E. Asia $+852-2698-7373$

Email:

edax.info-americas@ametek.com or edax.info-international@ametek.com Visit our website at wwwedax.com

\section{PHASE-CONTRAST IMAGING}

- Reveal the internal microstructure of a sample with resolutions below $200 \mathrm{~nm}$ 


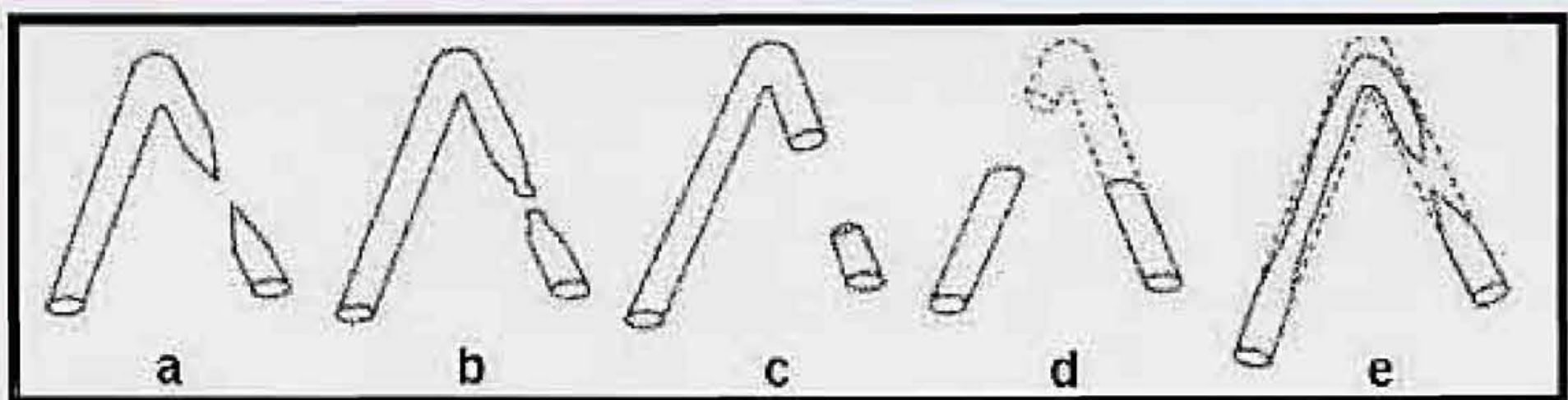

its central position.

No matter how an electron microscope is used the filament will eventually break giving the opportunity to analyze the filament and its ceramic. In the TEM, a normal break occurs to one side of the tip, the break should be between two tapered ends (Diagram 3 (a)). In the SEM we drive the filament much harder so that a normal break may well have blobs of metal at the point of failure (Diagram $3(b)$ ). Never panic with small blobs at the filament break in a SEM, if an operator is unlucky this can happen during saturation to the most experienced of us. In the TEM an overheated filament will break as above, except the ends may not taper, they will either be in the form of blobs of metal, or the blobs may have fallen off, leaving two blunt ends (Diagram 3 (c)). A similar situation may occur with SEM filaments. A filament blowing through high voltage discharge will have its end blown away, fortunately only seen in an unhappy TEM! (Diagram 3 (d)). A filament effected by a very poor vacuum will break due to severe oxidation. The filament will seem to break with the normal taper, but its life will have been very short. In this case the filament is very thin, far thinner than for a normal break (Diagram $3(\mathrm{e})$ ). Even if the gauges indicate the vacuum is good, this is not often a true representation of the actual gun vacuum, the ceramic will tell us even more.

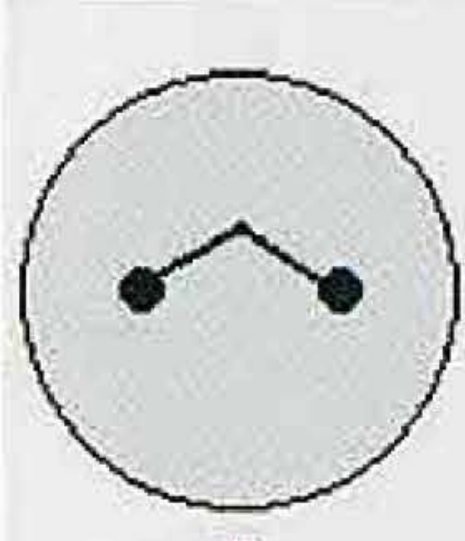

a

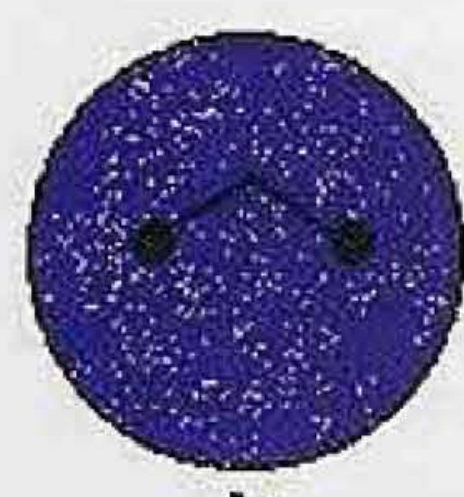

b

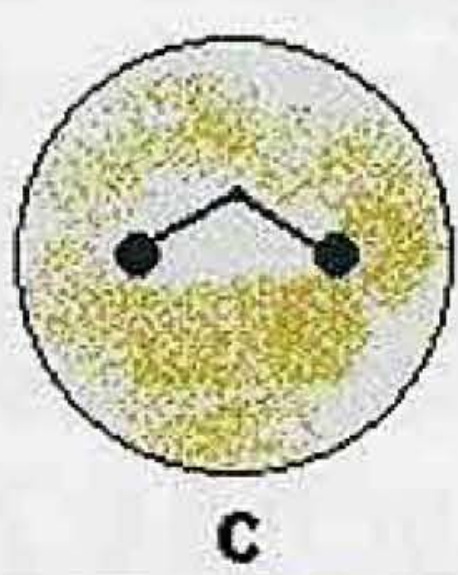

C
If the filament has been carefully saturated in a TEM, not overheated, the base will be a light blue in colour (Diagram 4 (a)). The colour comes from a light coating of evaporated tungsten. If the filament has been run very hard (typical of an SEM), either due to overheating in error, or due to the filament being placed very close to the cathode in order to obtain improved emission levels, the ceramic will be a dark blue in colour (Diagram 4 (b)). An orange to brown colouration is due to contamination, the filament had been operating in a poor vacuum environment (Diagram 4 (c)). This coloration would be expected if the filament had failed due to excess oxidation.

There are other reasons for a short filament life; namely that caused through poor quality wire being used to make the filament. From time to time the wire used to manufacture filaments is not up to standard, it contains a level of garbage; no criticism of the manufacturer of the filaments, more a criticism of the wire maker! In these circumstances, the filaments will thin normally but a good deal quicker and with far more "evaporated materials". (Diagram 5) demonstrates the build up of contaminants within the cathode due to poor filament quality. I have worked with several electron microscope manufacturers and every few years or so this type of problem crops up.

My advice to EM operators is NEVER use a complete box of filaments that have performed well; always save two. Then, in the situation that we now describe, one could pop in a filament from a

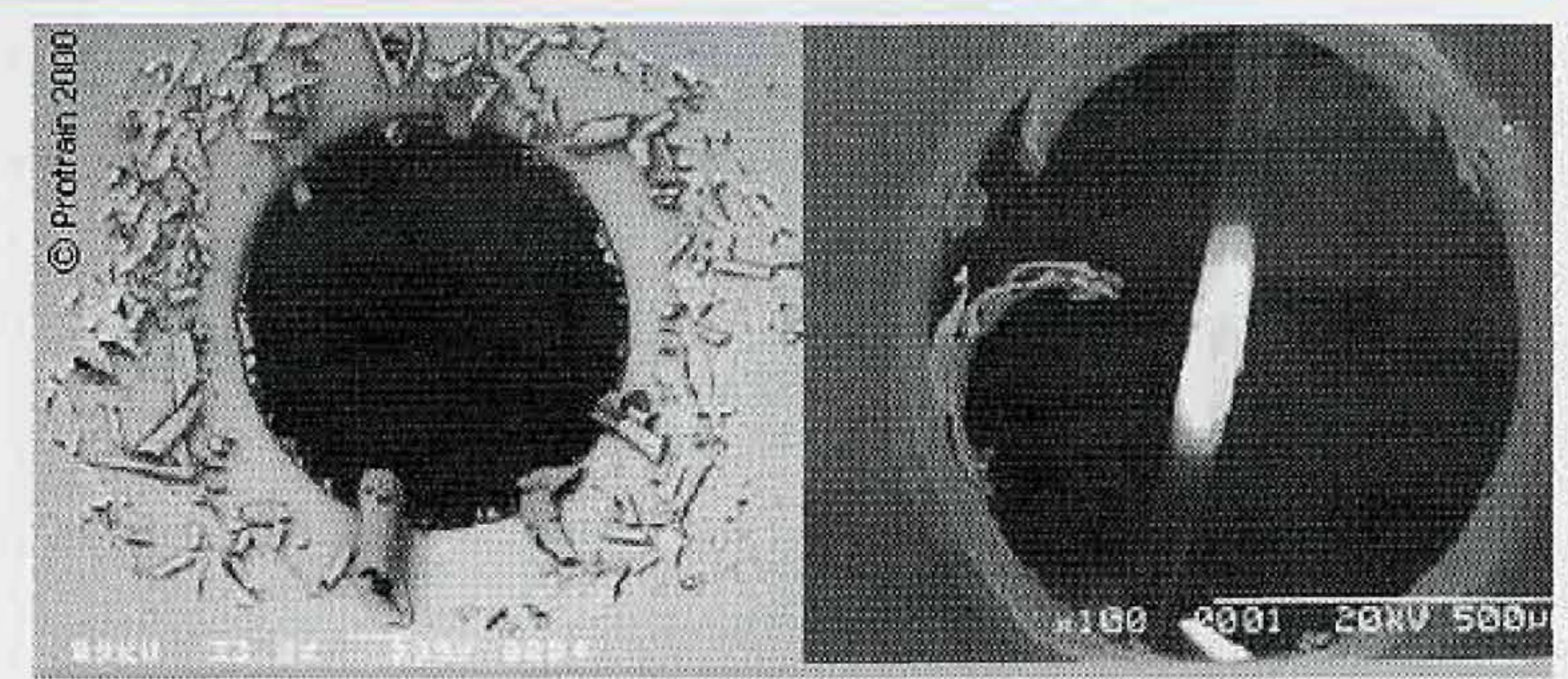

good box to prove either, that there is another problem, or that the new box of filaments is of a poor quality.

How to investigate -

1. Proceed with another filament from the new/problem box with great care, keep an eye on the operators to make sure that there is not an over saturation problem with one of them.

2. If the problem is repeated, replace with a filament from a good box.

3. If the problem continues there is probably a leak in the gun area, forget what the gauges say unless they are actually in the gun area.

4. If the problem went away it is proven that the new filaments are at error, return them to the supplier with an explanation of the problem.

5. Other areas of investigation if this does not solve the problem are-

a) The colour of the ceramic-yellow/brown suggests a vacuum leak.

b) A smelly gun chamber-smells of oil/ozone-suggests a vacuum leak.

c) Normal filament break but THE WHOLE OF THE FILAMENT HAS THINNED, not just the tip-gas attack-suggests a vacuum leak.

So there we are, in terms of the filament life: one gets what one pays for! To work with low levels of intensity in the SEM and TEM may be fine for some. However, if an operator wants to obtain more from a microscope, there will almost certainly be at a cost to pay-that cost would be filament life! 드

\section{Notes}

Steve Chapman has for 22 years run his own electron microscopy consultancy and training company: Protrain. he now works around the world running courses in SEM, TEM and EDS as well as offering consultancy on EM related subjects. His company also produce a range of interactive training CDs built on the training courses he runs worldwide.

\section{For only $\$ 3500$ for 5 days you could have a professional EM consultant provide SEM, TEM and EDS training, basic to advanced on site in your own laboratory on your own instruments, with consultancy tool}

For more information

WwW.emcourses.com

or email

protrain@emcourses.com 


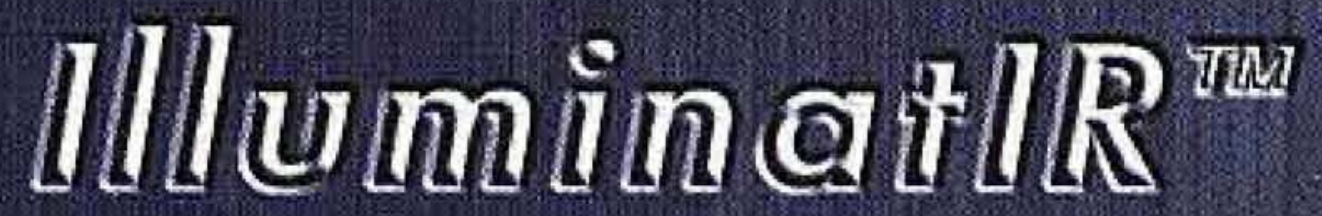

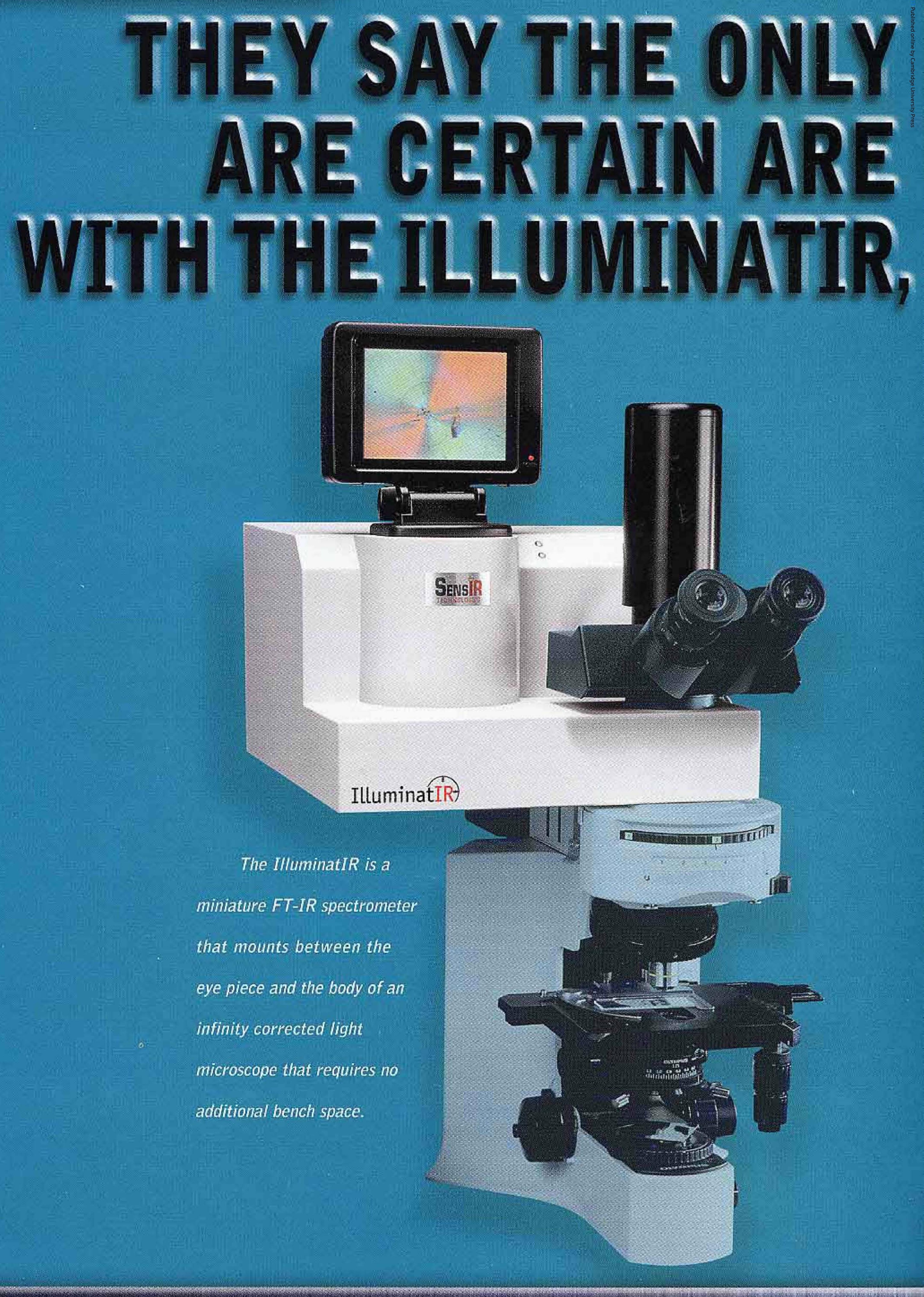




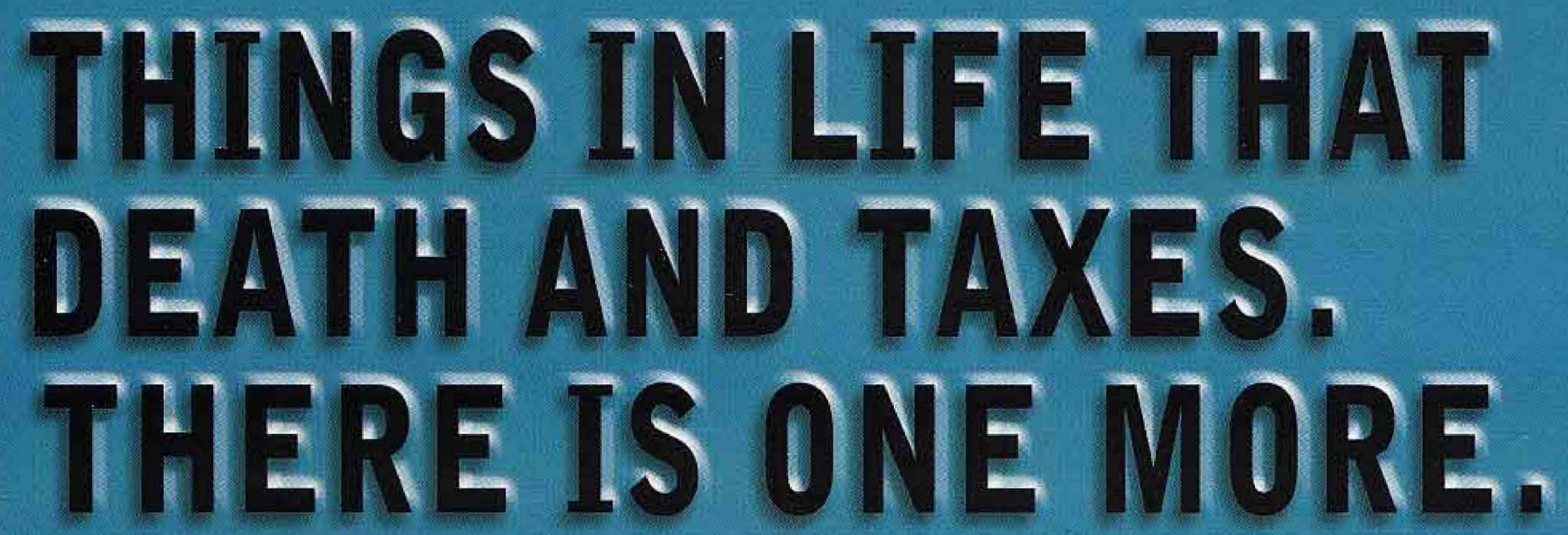

\section{IlluminatIR adds the power of FT-IR analysis to your light microscope}

Introducing the IlluminatIR, the only miniaturized FT-IR system that attaches directly to your favorite light microscope. Add the power of molecular analysis to your visual interpretation for the most accurate and objective results.
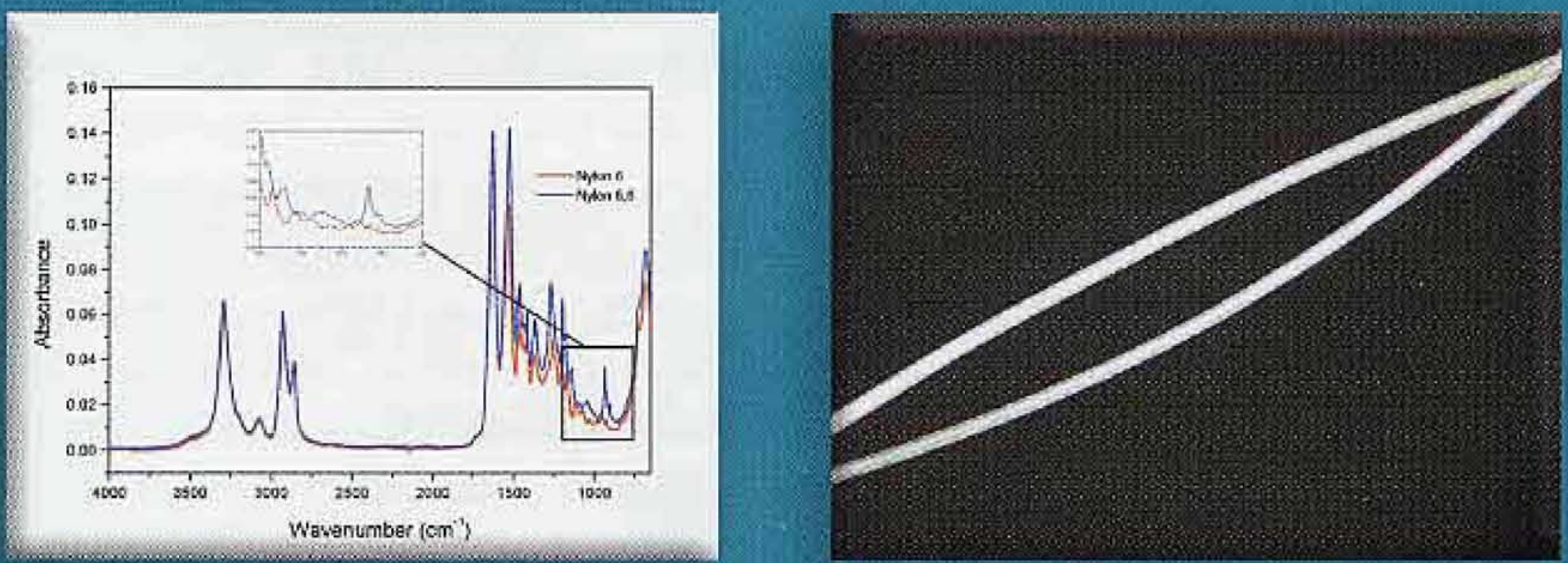

When samples are visually identical, FT-IR microscopy can show you the difference.

Visually these two nyion fibers are identical. However, when FT-IR analysis is run on each of the fibers and their spectra is overlaid the difference in the two fibers becomes clear. The top fiber was identiffed as Nylon 6 and the bottom fiber to be Nylon 6,6

Only IlluminatIR:

- provides high quality infrared data and superior visual quality

- easily attaches to infinity corrected microscopes

- maintains all the capabilities of your microscope,

i.e. polarization, fluorescence, Nomarski or image analysis, etc.

- can run FT-IR analysis simply with the turn of an objective - no need to move the sample

- adds these capabilities without taking up additional bench space

- very easy to use and maintain

The IlluminatIR: it will change the way microscopes are used forever.

\section{MAKE YOUR OWN OBJECTIVE OPINION.}

Call us to arrange a free demonstration of this breakthrough technology. 1-888-473-6747 Or visit our website www.sensir.com/objective

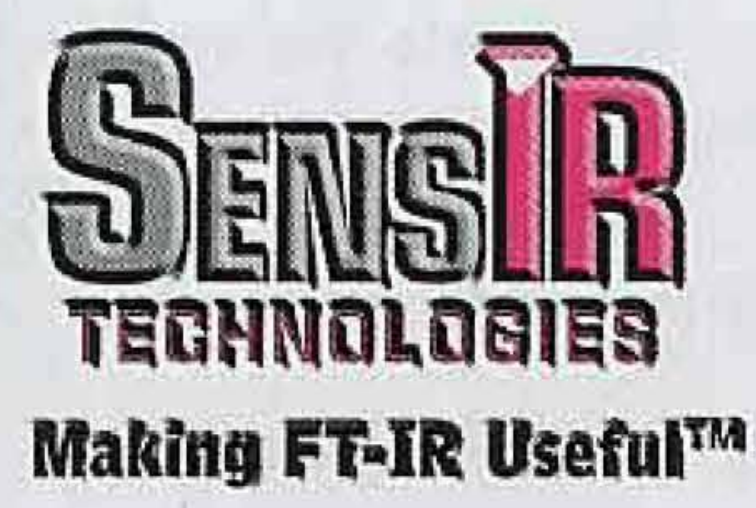

